\title{
UNSTABLE PRODUCTS OF SMOOTH CURVES
}

\author{
J. ROSS
}

\begin{abstract}
We give examples of smooth manifolds with negative first Chern class which are slope unstable with respect to certain polarisations, and so have Kähler classes that do not admit any constant scalar curvature Kähler metrics. These manifolds also have unstable Hilbert and Chow points. We compare this to the work of Song-Weinkove on the J-flow.
\end{abstract}

\section{INTRODUCTION}

A central problem in Kähler geometry is finding necessary and sufficient conditions for a Kähler class on a complex manifold $X$ to admit a constant scalar curvature Kähler ( $\mathrm{cscK})$ metric. It is well known that when the first Chern class of $X$ is negative, there exists a Kähler-Einstein (and hence $\operatorname{cscK}$ ) metric in $-c_{1}(X)$ $[1,22]$. Thus by a deformation argument due to LeBrun-Simanca [13] there exists a cscK metric in each class in an open neighborhood around $-c_{1}(X)$.

It has been an element of folklore that if $c_{1}(X)$ is negative then every Kähler class on $X$ admits a cscK metric. We prove in this paper that this is not the case by showing that this fails on the product of certain non-generic smooth curves.

The cscK problem is related to the stability of $X$. A conjecture of Yau states that a rational Kähler class $\Omega$ should admit a cscK metric if and only if the pair $(X, \Omega)$ is "stable" in the sense of geometric invariant theory [23]. The precise definition of stability, called K-stability, was introduced by Tian [19,20] and expanded by Donaldson [8]. One direction of the conjecture has essentially be proved: the existence of a cscK metric implies stability (see (2.1)).

To show that certain Kähler classes do not admit cscK metrics we shall use an obstruction for K-stability (and hence for cscK metrics) of Thomas and the author called slope stability $[16,17]$. The main results of this paper are the following:

Theorem 3.7. For $g \geq 5$ there exist smooth curves $C$ of genus $g$ such that $X=C \times C$ is not slope semistable with respect to certain polarisations. Thus there are Kähler classes on $X$ that do not admit Kähler metrics of constant scalar curvature.

Moreover this gives the first example of a manifold with negative first Chern class whose Hilbert and Chow points are unstable in the sense of geometric invariant theory.

Theorem 3.8. For $g \geq 5$ there exist smooth curves $C$ of genus $g$ such that $C \times C$ is not asymptotically Hilbert semistable (resp. not asymptotically Chow semistable) with respect to certain polarisations.

More specifically these results holds if $C$ admits a simple branched cover to $\mathbb{P}^{1}$ of degree $d$ with $2 \leq d-1<\sqrt{g}$ (3.3). Such curves are non-generic, since a general 
curve of genus $g$ admits a branched cover to $\mathbb{P}^{1}$ of degree

$$
d_{0}=\left[\frac{g+1}{2}\right]+1
$$

and none of degree $d<d_{0}$. ([10] p. 261).

Given a simple branched cover $\pi: C \rightarrow \mathbb{P}^{1}$ of degree $d$, consider the fibre product $C \times{ }_{\pi} C \subset X$. This contains the diagonal $\Delta$ and we let $Z$ be the residual divisor (i.e. $Z+\Delta=C \times{ }_{\pi} C$ ). We will show that if $2 \leq d-1<\sqrt{g}$ then $Z$ has negative self-intersection, and the "slope" of $Z$ as defined in Section 2 is less than that of $X$, which proves instability.

As suggested by Chen, such an example leads one to try and better understand the obstruction to finding cscK metrics in these classes. In particular one technique studied by Chen [3], Donaldson [7], Weinkove [21] and Song-Weinkove [18] is that of the J-flow. We comment in Section 4 as to what some of these results of [18] say when applied to products of curves.

Acknowledgments I would like to thank Richard Thomas for comments on the first draft of this paper, as well as continued encouragement. I also thank Xiuxiong Chen, Ian Morrison, Sean Paul, Michael Thaddeus and Ben Weinkove for useful conversations. Lazarsfeld's book [12] has been extremely helpful.

Notation By a polarisation on a variety $X$ we mean a choice of ample divisor which we usually denote by $L$ and will write $c_{1}(L)$ for the first Chern class of the associated line bundle. A $\mathbb{Q}$-divisor is a formal sum of divisors with rational coefficients, and an ample $\mathbb{Q}$-divisor is one which can be written as a sum of ample divisors (again with rational coefficients). The space of $\mathbb{Q}$-divisors modulo numerical equivalence is denoted $N^{1}(X)_{\mathbb{Q}}$. Abusing notation we will often not distinguish between a $\mathbb{Q}$-divisor and its class in $N^{1}(X)_{\mathbb{Q}}$.

\section{Slope Stability for VARieties}

The original link between K-stability and cscK metrics is due to Tian [19, 20]. For slope stability we require the definition of K-stability used by Donaldson [8] (the relation between the two definitions can be found in [15]).

Theorem 2.1. Fix a polarised manifold $(X, L)$. If there exists a constant scalar curvature Kähler metric in $c_{1}(L)$ then $(X, L)$ is K-semistable.

Proof. This is proved by in [6]. Alternatively one can use the existence of a cscK metric to show that the Mabuchi functional is bounded from below $[9,3]$ which in turn implies K-semistability [15].

The notion of slope stability for polarised varieties was introduced in $[16,17]$ as a necessary condition for K-stability. The general idea is that a non-generic subscheme of a polarised variety $(X, L)$ with certain numerical properties will have a slope that is too small, and this forces $(X, L)$ to be unstable.

Let $Z$ be a subscheme of a polarised variety $(X, L)$ and let $\pi: \widehat{X} \rightarrow X$ be the blowup of $X$ along $Z$ with exceptional divisor $E$. For sufficiently small positive $c$ the divisor $\pi^{*} L-c E$ is ample; so we can define the Seshadri constant of $Z$ as

$$
\epsilon(Z, L)=\sup \left\{c \in \mathbb{Q}: \pi^{*} L-c E \text { is ample }\right\} .
$$


Write the Hilbert polynomial of $L$ as $\chi(k L)=a_{0} k^{n}+a_{1} k^{n-1}+\cdots$ where $n=$ $\operatorname{dim} X$. For fixed $x \in \mathbb{Q}$ we define $a_{i}(x)$ by

$$
\chi\left(k\left(\pi^{*} L-x E\right)\right)=a_{0}(x) k^{n}+a_{1}(x) k^{n-1}+\cdots \quad \text { for all } k x \in \mathbb{N} .
$$

As $\chi\left(k \pi^{*} L-r E\right)$ is a polynomial in two variables of total degree at most $n$, we have that $a_{i}(x)$ is a polynomial and so extends to all real $x$. We let $\tilde{a}_{i}(x)=a_{i}-a_{i}(x)$ and for $0<c \leq \epsilon(Z, L)$ the slope of $X$ and $Z$ is defined to be $([16](3.1,3.14))$,

$$
\begin{aligned}
\mu(X, L) & =\frac{a_{1}}{a_{0}} \\
\mu_{c}\left(\mathcal{O}_{Z}, L\right) & =\frac{\int_{0}^{c} \tilde{a}_{1}(x)+\frac{\tilde{a}_{0}^{\prime}(x)}{2} d x}{\int_{0}^{c} \tilde{a}_{0}(x) d x},
\end{aligned}
$$

which are both finite (ibid. (4.21)).

Definition 2.2. We say that $(X, L)$ is slope semistable with respect to a subscheme $Z$ if

$$
\mu(X, L) \leq \mu_{c}\left(\mathcal{O}_{Z}, L\right) \quad \text { for all } 0<c \leq \epsilon(Z, L) .
$$

We say $(X, L)$ is slope semistable if it is slope semistable with respect to all subschemes.

Since the property of being slope semistable is invariant under replacing $L$ by some power (ibid. (3.10)), we extend the notion of slope semistability to ample $\mathbb{Q}$-divisors.

The definition of the slopes are made so that if $(X, L)$ is not slope semistable with respect to $Z$ then the degeneration to the normal cone of $Z$ prevents $(X, L)$ being K-semistable:

Theorem 2.3. If $(X, L)$ is not slope semistable then it is not K-semistable.

Proof. See [17] (4.18); when $X$ and $Z$ are smooth this is proved in [16] (4.2).

Slope stability for smooth surfaces. In this paper we will only consider the case that $X$ is a smooth surface and $Z$ is a curve. Then the blowup of $X$ along $Z$ is just $X$ itself, so

$$
\epsilon(Z, L)=\sup \{c \in \mathbb{Q}: L-c Z \text { is ample }\} .
$$

Letting $K$ be the canonical divisor of $X$, a simple application of the RiemannRoch theorem to calculate $a_{0}(x)$ and $a_{1}(x)$ yields ([16] (5.4)),

$$
\begin{aligned}
\mu(X, L) & =-\frac{K \cdot L}{L^{2}}, \\
\mu_{c}\left(\mathcal{O}_{Z}, L\right) & =\frac{3\left(2 L . Z-c\left(K . Z+Z^{2}\right)\right)}{2 c\left(3 L . Z-c Z^{2}\right)} .
\end{aligned}
$$

Notice that in this case $\epsilon(Z, L)$ as well as the slopes $\mu_{c}\left(\mathcal{O}_{Z}, L\right)$ and $\mu(X, L)$ depend only on the class of $L$ and $Z$ modulo numerical equivalence. We extend the equations (2.4) to any class $L$ in $N^{1}(X)_{\mathbb{Q}}$ which is not necessarily ample (in which case they may no longer be finite). 
Hilbert and Chow stability. Slope stability also gives an obstruction to the classical notions of stability for projective varieties. For $r \gg 0$ consider the embedding of $X$ via the linear series $|r L|$ into $\mathbb{P}^{N(r)}$. Up to change of change of coordinates, this determines a point $\operatorname{Hilb}\left(X, L^{r}\right)$ in the Hilbert scheme of $\mathbb{P}^{N(r)}$ (resp. a point $\operatorname{Chow}\left(X, L^{r}\right)$ in the Chow variety). The action of the automorphism group of $\mathbb{P}^{N(r)}$ induces a linearised action on the Hilbert scheme (resp. Chow variety), and one can apply the notions of geometric invariant theory to these spaces (see [14]).

Definition 2.5. We say that $(X, L)$ is asymptotically Hilbert (resp. Chow) semistable if for $r \gg 0$ the point $\operatorname{Hilb}\left(X, L^{r}\right)$ (resp. Chow $\left.\left(X, L^{r}\right)\right)$ is semistable in the sense of geometric invariant theory.

Theorem 2.6. If $(X, L)$ is not slope semistable then it is neither asymptotically Hilbert nor asymptotically Chow semistable.

Proof. The follows from (2.3) as asymptotic Hilbert (resp. Chow) semistability implies K-semistability ([17] (4.18)).

\section{Unstable Products of Curves}

We start with some standard material on the ample cone of products of curves, all of which can be found in [12]. Fix a smooth curve $C$ of genus $g \geq 2$ and let $X=C \times C$. If $\pi_{i}$ is the projection onto the $i$-th factor, and $p$ is a fixed point in $C$ then the class $f_{i}$ of the fibre $\pi_{i}^{-1}(p)$ in $N^{1}(X)_{\mathbb{Q}}$ is independent of $p$. The class of the canonical divisor of $X$ is $K=(2 g-2)\left(f_{1}+f_{2}\right)$ which is ample, so $X$ has negative first Chern class. Letting $\delta$ be the class of the diagonal we see that $f_{i}^{2}=0$, $f_{1} \cdot f_{2}=1, f_{i} . \delta=1$ and $\delta^{2}=2-2 g$.

Let $f=f_{1}+f_{2}$ and for convenience make the change of variables $\delta^{\prime}=\delta-f$. Then we have the following intersection numbers on $X$ :

$$
f^{2}=2, \quad \delta^{\prime} . f=0, \quad \text { and } \quad \delta^{\prime 2}=-2 g .
$$

Now consider the $\mathbb{Q}$-divisor

$$
L_{t}=t f-\delta^{\prime}
$$

which is ample for $t \gg 0$. We define

$$
s_{C}=\inf \left\{t: L_{t} \text { is ample }\right\} .
$$

Clearly $s_{C} \geq \sqrt{g}$ for if $L_{t}$ is ample then $0<L_{t}^{2}=2 t^{2}-2 g$. In fact conjecturally $s_{C}=\sqrt{g}$ for "most" curves (see Remark 4.5).

Recall that a branched cover from a curve to $\mathbb{P}^{1}$ is said to be simple if it has only ramifications which are locally $z \mapsto z^{2}$ and no two ramification points map to the same point in $\mathbb{P}^{1}$. Given a simple branched cover $\pi: C \rightarrow \mathbb{P}^{1}$ of degree $d$, consider the fibre product $C \times{ }_{\pi} C \subset X$. This contains the diagonal $\Delta$ and we let $Z$ be the residual divisor so $Z+\Delta=C \times{ }_{\pi} C$.

Lemma 3.1. The class of $Z$ in $N^{1}(X)_{\mathbb{Q}}$ is $(d-1) f-\delta^{\prime}$, so $Z$ has self-intersection $Z^{2}=2(d-1)^{2}-2 g$.

Proof. In the product $\mathbb{P}^{1} \times \mathbb{P}^{1}$ let $F_{i}, i=1,2$ be the numerical class of the coordinate planes and $D$ be the class of the diagonal. Then $D=F_{1}+F_{2}$ so

$$
Z+\delta=C \times{ }_{\pi} C=(\pi \times \pi)^{*}(D)=(\pi \times \pi)^{*}\left(F_{1}+F_{2}\right)=d f_{1}+d f_{2}=d f .
$$

Hence $Z=d f-\delta=(d-1) f-\delta^{\prime}$ as claimed. 
We will be interested in the case when $2 \leq d-1<\sqrt{g}$. Then $Z^{2}$ is negative, and we will show that $X$ is not slope semistable with respect to $Z$ for suitable polarisations.

Theorem 3.2 (Kouvidakis [11]). Suppose $C$ is a smooth curve of genus $g \geq 2$ which admits a simple branched cover to $\mathbb{P}^{1}$ of degree $d$ with $d-1 \leq \sqrt{g}$. Then $s_{C}=\frac{g}{d-1}$.

Proof. See [12] Theorem 1.5.8.

Theorem 3.3. Suppose $C$ is a smooth curve of genus $g$ which admits a simple branched cover $\pi: C \rightarrow \mathbb{P}^{1}$ of degree $d$ with $2 \leq d-1<\sqrt{g}$.

Then $X=C \times C$ is not slope semistable with respect $L_{t}$ for $t$ sufficiently close to $s_{C}$.

Proof. Let $t>s_{C}$ so $L_{t}$ is ample. The canonical divisor of $X$ is $K=(2 g-2) f$ so from $(2.4)$

$$
\mu\left(X, L_{t}\right)=-\frac{K \cdot L_{t}}{L_{t}^{2}}=-\frac{t(2 g-2)}{t^{2}-g} .
$$

By (3.2), $s_{C}=\frac{g}{d-1}$. Letting $Z$ be the curve from Lemma 3.1 whose class is $(d-1) f-\delta^{\prime}$ we now bound the Seshadri constant of $Z$. Since

$$
t-(d-1)>s_{C}-(d-1)=\frac{g}{d-1}-(d-1)>0
$$

we have that $L_{t}-Z=t f-\delta^{\prime}-\left((d-1) f-\delta^{\prime}\right)=(t-(d-1)) f$ is ample. Thus $\epsilon\left(Z, L_{t}\right) \geq 1$.

To calculate the slope of $Z$ we need the quantities

$$
\begin{aligned}
L_{t} . Z & =\left(t f-\delta^{\prime}\right) \cdot\left((d-1) f-\delta^{\prime}\right) \\
& =2 t(d-1)-2 g, \\
K . Z & =(2 g-2) f \cdot\left((d-1) f-\delta^{\prime}\right)=2(2 g-2)(d-1), \\
Z^{2} & =\left((d-1) f-\delta^{\prime}\right)^{2}=2(d-1)^{2}-2 g .
\end{aligned}
$$

Thus from (2.4),

$$
\begin{aligned}
\mu_{1}\left(\mathcal{O}_{Z}, L_{t}\right) & =\frac{3\left(2 L_{t} \cdot Z-\left(K . Z+Z^{2}\right)\right)}{2\left(3 L_{t} \cdot Z-Z^{2}\right)} \\
& =\frac{3\left(4 t(d-1)-4 g-2(2 g-2)(d-1)-2(d-1)^{2}+2 g\right)}{2\left(6 t(d-1)-6 g-2(d-1)^{2}+2 g\right)} .
\end{aligned}
$$

We claim that $\mu_{1}\left(\mathcal{O}_{Z}, L_{t}\right)<\mu\left(X, L_{t}\right)$ as $t$ tends to $s_{C}=\frac{g}{d-1}$ from above. Since this is an open condition it is sufficient to show that it holds when $t=s_{C}$. By (3.4, $3.6)$,

$$
\begin{aligned}
\mu\left(X, L_{s_{C}}\right) & =-\frac{(2 g-2)(d-1)}{g-(d-1)^{2}} \\
\mu_{1}\left(\mathcal{O}_{Z}, L_{s_{C}}\right) & =\frac{3\left(g-(2 g-2)(d-1)-(d-1)^{2}\right)}{2\left(g-(d-1)^{2}\right)}
\end{aligned}
$$


(notice that our assumption $d-1<\sqrt{g}$ ensures that both of these are finite). Hence as $d-1 \geq 2$,

$$
\begin{aligned}
2\left(g-(d-1)^{2}\right) & \cdot\left(\mu_{1}\left(\mathcal{O}_{Z}, L_{s_{C}}\right)-\mu\left(X, L_{s_{C}}\right)\right) \\
& =3 g-3(2 g-2)(d-1)-3(d-1)^{2}+2(2 g-2)(d-1) \\
& =3 g-(2 g-2)(d-1)-3(d-1)^{2} \\
& \leq 3 g-2(2 g-2)-12<0 .
\end{aligned}
$$

Thus $\epsilon\left(Z, L_{t}\right) \geq 1$ and $\mu_{1}\left(\mathcal{O}_{Z}, L_{t}\right)<\mu\left(X, L_{t}\right)$ as $t$ tends to $s_{C}$ from above, which proves that $\left(X, L_{t}\right)$ is not slope semistable.

Theorem 3.7. For $g \geq 5$ there exist smooth curves $C$ of genus $g$ such that $X=$ $C \times C$ is not slope semistable with respect to certain polarisations. Thus there are Kähler classes on $X$ that do not admit Kähler metrics of constant scalar curvature.

Proof. By the Riemann existence theorem there exist smooth curves of genus $g$ which admit a simple branched covering over $\mathbb{P}^{1}$ of degree $2 \leq d-1<\sqrt{g}$ (in fact one can even take $d=3$ ). So by (3.3), $X=C \times C$ is not slope stable with respect to certain polarisations and thus not K-semistable by (2.3). The application to cscK metrics comes from (2.1).

Theorem 3.8. For $g \geq 5$ there exist smooth curves $C$ of genus $g$ such that $X=C \times C$ is not asymptotically Hilbert semistable (resp. not asymptotically Chow semistable) with respect to suitable polarisations.

Proof. This follows from (3.7) and (2.6).

Remark 3.9. Let $\left(X_{i}, L_{i}\right) i=1,2$ be polarised manifolds and $\pi_{i}: X_{1} \times X_{2} \rightarrow X_{i}$ be the projection maps. If $L=\pi_{1}^{*} L_{1}+\pi_{2}^{*} L_{2}$ then

$$
\mu\left(X_{1} \times X_{2}, L\right)=\mu\left(X_{1}, L_{1}\right)+\mu\left(X_{2}, L_{2}\right) .
$$

Moreover if $Z$ is subscheme of $X_{1}$ then one can calculate the slope of $Z \times X_{2} \subset$ $X_{1} \times X_{2}$ is $\mu_{c}\left(\mathcal{O}_{Z \times X_{1}}, L\right)=\mu_{c}\left(\mathcal{O}_{Z}, L_{1}\right)+\mu\left(X_{2}, L_{2}\right)$. Thus if $\left(X_{1}, L_{1}\right)$ is slope unstable so is the product $\left(X_{1} \times X_{2}, L\right)$.

So by taking the product of an slope unstable surface with any manifold with negative first Chern class, we get manifolds of any dimension $n \geq 2$ with negative first Chern class which have Kähler classes that do not admit cscK metrics.

\section{The J-FLOW ON PRODUCTS OF CURVES}

The Mabuchi functional for a given Kähler class $\Omega$ on a complex manifold $X$ has as its critical points the metrics which are cscK. Conjecturally the existence of a $\operatorname{cscK}$ metric in $\Omega$ is equivalent to the properness of the Mabuchi functional. This is known to be true when $\Omega$ is proportional to the canonical class [2, 20]; and when $\Omega$ admits a cscK metric the Mabuchi functional is necessarily bounded from below $[4,9]$

Now suppose that $X$ has negative first Chern class. Chen [3] introduces a flow on Kähler manifolds, called the J-flow, and points out that convergence of this flow implies lower boundedness of the Mabuchi functional. In [21] it is shown that on a surface with negative first Chern class, the J-flow converges as long as the class $-2\left(\int_{X} c_{1}(X) . \Omega\right) \Omega+\left(\int_{X} \Omega^{2}\right) c_{1}(X)$ is positive. 
The following theorem is a particular case of Theorems 1.2 and 1.4 in [18] applied to polarised surfaces.

Theorem 4.1. Let $(X, L)$ be a polarised surface and define a divisor by

$$
\alpha=2(K . L) L-\left(L^{2}\right) K .
$$

- If $\alpha$ is ample then the J-flow converges and the Mabuchi functional is proper on the class $c_{1}(L)$.

- If $\alpha$ is not ample then there exist $m$ irreducible curves $E_{i}$ of negative self intersection and positive numbers $a_{i}$ such that

$$
\alpha-\sum_{i=1}^{m} a_{i} E_{i} \text { is ample. }
$$

In fact if $\alpha$ is not ample then the J-flow "blows up" along the intersection of all divisors in the linear series $\left|\Sigma a_{i} E_{i}\right|[18]$.

From this theorem one might expect that $(X, L)$ is stable if $\alpha$ is ample, and perhaps that if $\alpha$ is not ample that the $E_{i}$ witness instability of $(X, L)$ (c.f. [18] Remark 4.7).

When $C$ is a curve, $X=C \times C$ and $L=L_{t}=t f-\delta^{\prime}$ with $t>s_{C}$ it is easy to determine when $\alpha$ is ample.

Lemma 4.3. Let $L=L_{t}=t f-\delta^{\prime}$ with $t>s_{C}$. Then $\alpha=2(K . L) L-\left(L^{2}\right) K$ is ample if and only if $t^{2}+g>2 t s_{C}$ if and only if $t>s_{C}+\sqrt{s_{C}^{2}-g}$.

Proof. As $L_{t}^{2}=2 t^{2}-2 g$ and $K . L_{t}=2 t(2 g-2)$ we have

$$
\begin{aligned}
\alpha & =4 t(2 g-2)\left(t f-\delta^{\prime}\right)-\left(2 t^{2}-2 g\right)(2 g-2) f \\
& =2(2 g-2)\left(\left(t^{2}+g\right) f-2 t \delta^{\prime}\right)
\end{aligned}
$$

which is ample if and only if $t^{2}+g>2 t s_{C}$ which occurs if and only if $h(t)=$ $t^{2}-2 t s_{C}+g>0$. But the roots of $h$ are $s_{C} \pm \sqrt{s_{C}^{2}-g}$ and $s_{C} \geq \sqrt{g}$ so the lemma follows.

Thus one can deduce properness of the Mabuchi functional when $s_{C}=\sqrt{g}$.

Corollary 4.4. If $s_{C}=\sqrt{g}$ then the Mabuchi function is proper on any class on $X=C \times C$ of the form $c_{1}\left(L_{t}\right)$ for $t>s_{C}$.

Remark 4.5. If $g$ is a perfect square and $C$ is very general curve of genus $g$ then $s_{C}=\sqrt{g}$ ([12] Corollary 1.5.9). If the Nagata conjecture holds and $g \geq 10$ then the same conclusion holds without the hypothesis that $g$ is a perfect square [5].

Remark 4.6. Let $C$ be as in (3.3) and $X=C \times C$. It would be interesting to know if the Mabuchi functional is bounded on the class $c_{1}\left(L_{t}\right)$ if and only if $t>s_{C}+\sqrt{s_{C}^{2}-g}$.

It is not the case that the curve $Z=(d-1) f-\delta^{\prime}$ from (3.3) always slope destabilises when $t<s_{C}+\sqrt{s_{C}^{2}-g}$. For example let $C$ be a curve of genus $g=5$ admitting a simple branched cover to $\mathbb{P}^{1}$ of degree $d=3$. Then $s_{C}=5 / 2$ so $s_{C}+\sqrt{s_{C}^{2}-g}=\frac{5+\sqrt{5}}{2}>3$. Put $t=3$ so $L_{3}=3 f-\delta^{\prime}$ is ample. The slope of $Z$ is

$$
\mu_{c}\left(\mathcal{O}_{Z}, L\right)=\frac{3(2-15 c)}{2 c(3+c)}
$$

which is greater than $\mu(X, L)=-6$ for all $c>0$. 
By the previous lemma $\alpha$ is not ample. It is unfortunately not the case that any divisor satisfying (4.2) necessarily destabilise (c.f. [18] Remark 4.7) because in the example above, $\alpha-33 Z=158 f-63 \delta^{\prime}$ is ample.

\section{REFERENCES}

[1] T. Aubin. Équations du type Monge-Ampère sur les variétés kähleriennes compactes. $C$. $R$. Acad. Sci. Paris Sér. A-B, 283(3):Aiii, A119-A121, 1976.

[2] S. Bando and T. Mabuchi. Uniqueness of Einstein Kähler metrics modulo connected group actions. In Algebraic geometry, Sendai, 1985, volume 10 of Adv. Stud. Pure Math., pages 11-40. North-Holland, 1987.

[3] X.X. Chen. On the lower bound of the Mabuchi energy and its application. Internat. Math. Res. Notices, (12):607-623, 2000.

[4] X.X. Chen and G. Tian. Geometry of Kähler metrics and holomorphic foliation by discs. math.DG/0409433.

[5] C. Ciliberto and A. Kouvidakis. On the symmetric product of a curve with general moduli. Geom. Dedicata, 78(3):327-343, 1999.

[6] S. Donaldson. Lower bounds on the calabi functional. math.DG/0506501.

[7] S. Donaldson. Moment maps and diffeomorphisms. Asian J. Math., 3(1):1-15, 1999. Sir Michael Atiyah: a great mathematician of the twentieth century.

[8] S. Donaldson. Scalar curvature and stability of toric varieties. Journal of Differential Geometry, 62:289-349, 2002.

[9] S. Donaldson. Scalar curvature and projective embeddings, II. The Quaterly Journal of Mathematics, 2005.

[10] P. Griffiths and J. Harris. Principles of Algebraic Geometry. Wiley Classics Library. Wiley, 1978.

[11] A. Kouvidakis. Divisors on symmetric products of curves. Trans. Amer. Math. Soc., 337(1):117-128, 1993.

[12] R. Lazarsfeld. Positivity in algebraic geometry. I. Ergebnisse der Mathematik und ihrer Grenzgebiete. 3. Folge.

[13] C. LeBrun and S. R. Simanca. Extremal Kähler metrics and complex deformation theory. Geom. Funct. Anal., 4(3):298-336, 1994.

[14] D. Mumford. Stability of projective varieties. Enseignement Mathmatique (2), 23(1-2):39110, 1977.

[15] S. Paul and G. Tian. Analysis of geometric stability. Int. Math. Res. Not., (48):2555-2591, 2004.

[16] J. Ross and R. P. Thomas. An obstruction to the existence of constant scalar curvature Kähler metrics. math.DG/0412518.

[17] J. Ross and R. P. Thomas. A study of the Hilbert-Mumford criterion for the stability of projective varieties. math.AG/0412519.

[18] J. Song and B. Weinkove. On the convergence and singularities of the J-flow with applications to the mabuchi energy. math.DG/0410418.

[19] G. Tian. The K-energy on hypersurfaces and stability. Comm. Anal. Geom., 2(2):239-265, 1994.

[20] G. Tian. Kähler-Einstein metrics with positive scalar curvature. Invent. Math., 130(1):1-37, 1997.

[21] B. Weinkove. On the J-flow in higher dimensions and the lower boundedness of the Mabuchi energy. math.DG/0309404.

[22] S. T Yau. On the Ricci curvature of a compact Kähler manifold and the complex MongeAmpère equation. I. Comm. Pure Appl. Math., 31(3):339-411, 1978.

[23] S. T. Yau. Open problems in geometry. In Differential geometry: partial differential equations on manifolds (Los Angeles, CA, 1990), volume 54 of Proc. Sympos. Pure Math., pages 1-28. Amer. Math. Soc., Providence, RI, 1993.

jaross@math.columbia.edu

Department of Mathematics, Columbia University, New York, NY 10027. USA. 